\title{
LIMITAÇÕES DAS REANÁLISES PARA ALTAS LATITUDES DO HEMISFÉRIO SUL
}

\author{
ALBERTO SETZER E MARY KAYANO \\ Centro de Previsão de Tempo e Estudos Climáticos, Instituto Nacional de Pesquisas Espaciais, \\ (CPTEC/INPE) - São José dos Campos - SP, Brasil \\ alberto.setzer@cptec.inpe.br, mary.kayano@cptec.inpe.br \\ Recebido Setembro 2007 - Aceito Março 2009 \\ RESUMO
}

\begin{abstract}
Apresenta-se uma comparação de resultados de reanálise para temperatura do ar em $1000 \mathrm{hPa}$ e pressão reduzida ao nível do mar nas latitudes altas do hemisfério sul, no período 1979-2000. O estudo usa três conjuntos de reanálises, NCEP-NCAR 1 e 2, e ECMWF/ERA-40, e as diferenças entre os mesmos tornam-se significativas e aumentam a partir da latitude de $\sim 60^{\circ}$ sul, sendo particularmente altas sobre a região continental antártica. Considerando a média dos 22 anos, a diferença da temperatura entre as reanálises nos casos extremos chegou a $12^{\circ} \mathrm{C}$ no outono (Março, Abril e Maio) e inverno (Junho, Julho e Agosto), e a da pressão reduzida, a $30 \mathrm{hPa}$, nestes mesmos períodos. Na região costeira antártica, a comparação com dados de duas estações meteorológicas de superfície indicou diferenças entre as temperaturas médias sazonais medidas e as das reanálises. A estação mais ao norte, Bellinghausen $\left(62^{\circ} 12^{\prime} \mathrm{S}\right.$ e $\left.058^{\circ} 56^{\prime} \mathrm{W}\right)$, apresentou diferenças menores, de no máximo $1.8^{\circ} \mathrm{C}$, e a de Mirny $\left(66^{\circ} 33^{\prime} \mathrm{S}\right.$ e $\left.093^{\circ} 01^{\prime} \mathrm{E}\right)$ de até $4.5^{\circ} \mathrm{C}$, em conformidade com o aumento constatado das diferenças segundo a latitude. Como possível causa das diferenças entre as reanálises, e entre elas e os dados medidos, sugere-se: falhas no controle de qualidade dos dados medidos na região antártica, e; o fato da atmosfera antártica, particularmente nos casos de frio extremo, diferir da atmosfera padrão usada globalmente para a redução de pressão e temperatura a outros níveis da atmosfera. A magnitude das diferenças constatadas tem o potencial de prejudicar as séries temporais meteorológicas e a determinação de variações climáticas antárticas. Palavras-chave: Antártica; reanálise; climatologia.
\end{abstract}

\begin{abstract}
LIMITATIONS OF REANALYSES FOR HIGH LATITUDES OF THE SOUTHERN HEMISPHERE.

This paper presents a comparison of reanalyses results for air temperature at $1000 \mathrm{hPa}$ and atmospheric pressure reduced to sea level at the high latitudes of the southern hemisphere, during the period of 1979-2000. The data used comprised three sets of reanalyses, NCEP-NCAR 1 and 2, and ECMWF/ ERA-40. The differences between them become significant and increase from $\sim 60^{\circ}$ south, reaching particularly high values at the center of the Antarctic continent. Considering the average for the 22 years, the difference in temperature for the reanalyses in the extreme cases reached up to $12^{\circ} \mathrm{C}$ in the autumn (March, April and May), and winter (June, July and (August), and for the reduced pressure, up to $30 \mathrm{hPa}$, in these same seasons. In the coastal Antarctic region the comparison with data from two surface weather stations indicated differences between the average seasonal temperatures actually measured, and those of the reanalyses. The most northern station, Bellinghausen $\left(62^{\circ} 12^{\prime} \mathrm{S}\right.$ e $\left.058^{\circ} 56^{\prime} \mathrm{W}\right)$, presented smaller differences, up to $1.8^{\circ} \mathrm{C}$, and Mirny $\left(66^{\circ} 33^{\prime} \mathrm{S}\right.$ e $\left.093^{\circ} 01^{\prime} \mathrm{E}\right)$, up to $4.5^{\circ} \mathrm{C}$; this is in agreement with the herein proposed increase of the differences according to the latitude. As a possible explanation for these differences among sets of reanalyses and between them and the measured surface data, we suggest: faulty quality control of the input data from Antarctica, and; the difference between the Antarctic atmosphere, particularly in the cases of extreme cold, with that of the standard atmosphere that is used globally to reduce pressure and temperature to other levels in the atmosphere. The magnitude of the differences reported has the potential to hamper the study of temporal series of meteorological data and of climatic variations for Antarctica
\end{abstract}

Keywords: Antactica; Reanalyses; Climatology 


\section{INTRODUÇÃO}

Questionamentos sobre a qualidade dos dados de reanálise para altas latitudes do Hemisfério Sul têm sido levantados nos últimos anos. Hines et al. (2000) compararam os dados da Reanálise do NCEP-NCAR (NNR) para o período 1949-1998 com séries de estações de superfície na Antártica. Eles concluíram que variações de longo prazo, mostrando um decréscimo $0.2 \mathrm{hPa} /$ ano na pressão em superfície a $65^{\circ} \mathrm{S}$, não tiveram correspondente queda na série de estações meteorológicas; tendências de longo prazo erradas foram encontradas ao sul de $45^{\circ} \mathrm{S}$, e variações de curto prazo foram consideradas adequadas somente após 1970. Marshall e Harangozo (2000) chegaram a conclusões similares para o Oceano Pacífico Sul. Marshall (2003) investigou tendências no modo anular do Hemisfério Sul (SAM) para décadas recentes usando dados do NNR e afirmou que "...erros nos dados do NNR tem o potencial de invalidar os resultados publicados sobre mudanças no SAM". Esta colocação resultou da afirmação que "...tendências calculadas com a diferença entre $40^{\circ} \mathrm{S}$ e $65^{\circ} \mathrm{S}$ da pressão ao nível médio do mar (PNM) e o SAM estão exageradas por um fator de 3 e 2, respectivamente no NNR.". Marshall (2003) também indicou que os dados de reanálise do ECWMF/ERA-40 “...podem ser usados com alta confiança ao menos desde 1973". A questão das tendências na pressão no SAM no período de 1958-2000 foi ainda examinada por Marshall el al. (2004), que demonstraram que “...recentes tendências observadas no SAM anual e de verão austral são improváveis de ocorrer devido à variabilidade climática interna." Bromwich e Fogt (2004) documentaram a melhora das reanálises antárticas com o uso de satélites meteorológicos a partir do final da década de 1970; embora a correlação entre as reanálises tenha melhorado, a existência de "grandes discrepâncias" é indicada, em particular nos invernos, como provável conseqüência de esquemas distintos de assimilação de dados e da diminuição de dados de estações nestes períodos. Mais recentemente, em um workshop sobre as reanálises do NNR (versões 1 e 2), ECMWF/ERA-40 e JRA-25 japonês organizado pelo Scientific Committee on Antarctic Research (SCAR, 2006), as conclusões foram que “...enquanto estas reanálises são extremamente valiosas, todas tiveram ou têm falhas sérias", e que "...durante a moderna era dos satélites existem grandes diferenças nas reanálises". Este workshop foi também documentado por Bromwich et al. (2007), cuja conclusão aponta para resultados positivos do uso das reanálises antárticas, apesar de suas limitações. Incoerências nas séries históricas das climatologias antárticas geradas com dados de reanálise também foram indicadas por Setzer et al.(2006).

Acima foram transcritos alguns trechos de trabalhos anteriores que alertam para as limitações dos conjuntos de dados de reanálises. O presente trabalho tem como objetivo apresentar evidências das discrepâncias entre reanálises das altas latitudes do hemisfério sul, com uma primeira indicação de sua magnitude.

\section{DADOS E METODOLOGIA}

As seguintes fontes de dados de reanálise foram usadas para obter séries climáticas do hemisfério sul: reanálise 1 do NCEP-NCAR 1, NNR-1 (Kalnay et al., 1996); reanálise 2 do NCEP-NCAR 2, NNR-2 (Kanamitsu et al., 2002), e; reanálise do ECMWF/ERA-40, ERA-40 (Uppala et al., 2005).

Os dados do NNR-1 e NNR-2 foram fornecidos pela NOAA/OAR/ESRL PSD, Boulder, Colorado, USA, por meio do sitio http://www.cdc.noaa.gov/. Os dados do ERA-40 usados neste estudo foram obtidos da servidora de dados do ECMWF, no endereço http://www.ecmwf.int. Dados reanalisados mensais de PNM e de temperatura do ar em $1000 \mathrm{hPa}$ para o período de 1979 a 2001, foram obtidos destas três fontes de dados, na resolução de $2.5^{\circ} \mathrm{em}$ latitude e longitude.

Os dados foram analisados para a faixa global entre $50^{\circ} \mathrm{S}$ e $90^{\circ} \mathrm{S}$, e as diferenças entre as três reanálises estão apresentadas. Os dados foram considerados por estação do ano, sendo: o verão, com os meses de dezembro, janeiro e fevereiro; o outono, com março, abril e maio; o inverno, com junho, julho e agosto, e; a primavera, com setembro, outubro e novembro. O período de análise de 1979 a 2001, em princípio exclui os dados mais inconsistentes, de antes do advento do monitoramento com satélites. As séries de estações de superfície na Antártica são as disponíveis no SCAR-BAS-READER (2006); foram utilizadas as médias mensais, por sua vez obtidas a partir dos dados registrados nos horários sinóticos convencionais a cada três horas.

Foi feita a avaliação da significância estatística da diferença das médias (climatologias), na qual o número de graus de liberdade usado foi o do número de anos. Sendo $X_{1} \mathrm{e} X_{2}$ duas variáveis com $n_{1}$ e $n_{2}$ e valores, $\mathrm{S}_{1}$ e $\mathrm{S}_{2}$ desvios padrão, e suas médias indicadas por $\bar{X}_{1} \mathrm{e} \bar{X}_{2}$, respectivamente, admite-se que a diferença $X_{1}-X_{2}$ tem a distribuição " $\mathrm{t}$ " de Student. Considerase que o valor absoluto da diferença, $\bar{X}_{1}-\bar{X}_{2}$, é estatisticamente significante se exceder o valor limítrofe de (Press et al., 1986):

$$
t_{\alpha(n 1+n 2-2)} \sqrt{\left(n_{1}-1\right) S_{1}^{2}+\left(n_{2}-1\right) S_{2}^{2}} \sqrt{\frac{n_{1}+n_{2}}{n_{1} n_{2}\left(n_{1}+n_{2}-2\right)}}
$$

Os dados nas Figuras 1 e 2 seguiram o critério acima, e apresentam o nível de confiança de 95\%.

\section{RESULTADOS E DISCUSSÃO}

A Figura 1a mostra as diferenças na temperatura do ar em $1000 \mathrm{hPa}$ entre o ERA-40 e o NNR-1, variando no melhor 
caso de $3^{\circ} \mathrm{C}$ para o verão nas longitudes da Antártica continental de 0 a $70^{\circ} \mathrm{E}$, para a pior condição de $15^{\circ} \mathrm{C}$ durante o inverno, aproximadamente em $80^{\circ} \mathrm{S}$ e $20^{\circ} \mathrm{E}$. A Figura $1 \mathrm{~b}$ mostra o mesmo tipo de comparação, mas para o ERA-40 e o NNR-2, e onde também podem ser notadas diferenças significantes, de $3^{\circ} \mathrm{C}$ no inverno e primavera a $6^{\circ} \mathrm{C}$ no verão e outono. As diferenças entre o NNR-1 e NNR-2 variam de um máximo de $9^{\circ} \mathrm{C}$ no outono e inverno, a um mínimo de $-3^{\circ} \mathrm{C}$ no verão (Figura $1 \mathrm{c}$ ). Para latitudes antárticas na linha da costa, as diferenças em temperatura para os três conjuntos de dados são normalmente pequenas e tendem a zero em aproximadamente $60^{\circ} \mathrm{S}$, e mais ao norte.

As diferenças para PNM são mostradas na Figura 2. Discrepâncias de até $40 \mathrm{hPa}$ ocorreram na Antártica continental durante o inverno, quando os dados do ERA-40 e NNR-1 foram comparados em $85^{\circ} \mathrm{S}$ e $70^{\circ} \mathrm{E}$ (Figura 2a). Considerando-se o ERA-40 e NNR-2, a diferença é reduzida para um máximo de $20 \mathrm{hPa}$ no inverno (Figura 2b); para o NNR-1 e NNR-2, como visto na Figura 2c, a máxima diferença é de aproximadamente $20 \mathrm{hPa}$, também no inverno. Como no caso das temperaturas, todas as discrepâncias tendem a desaparecer para latitudes menores, e em 600 S são mínimas. Erros maiores nas reanálises para os períodos de inverno também foram apontadas em estudos anteriores, como em Bromwich e Fogt (2004).

Outras variáveis foram também comparadas nas reanálises, porém os resultados não estão apresentados neste texto. Por exemplo, dependendo da reanálise, os campos de anomalias de geopotencial em $500 \mathrm{hPa}$ indicam padrões regionais distintos, inclusive com sinais opostos. A pressão atmosférica e a temperatura são utilizadas nos cálculos de outras variáveis, como a altura geopotencial, temperatura potencial, umidade absoluta, etc., que por sua vez conseqüentemente, também apresentarão discrepâncias entre os conjuntos de reanálises.

Dados de estações meteorológicas de superfície na Antártica confirmaram as discrepâncias entre as reanálises

a) ERA-40 minus NNR-1 T1000
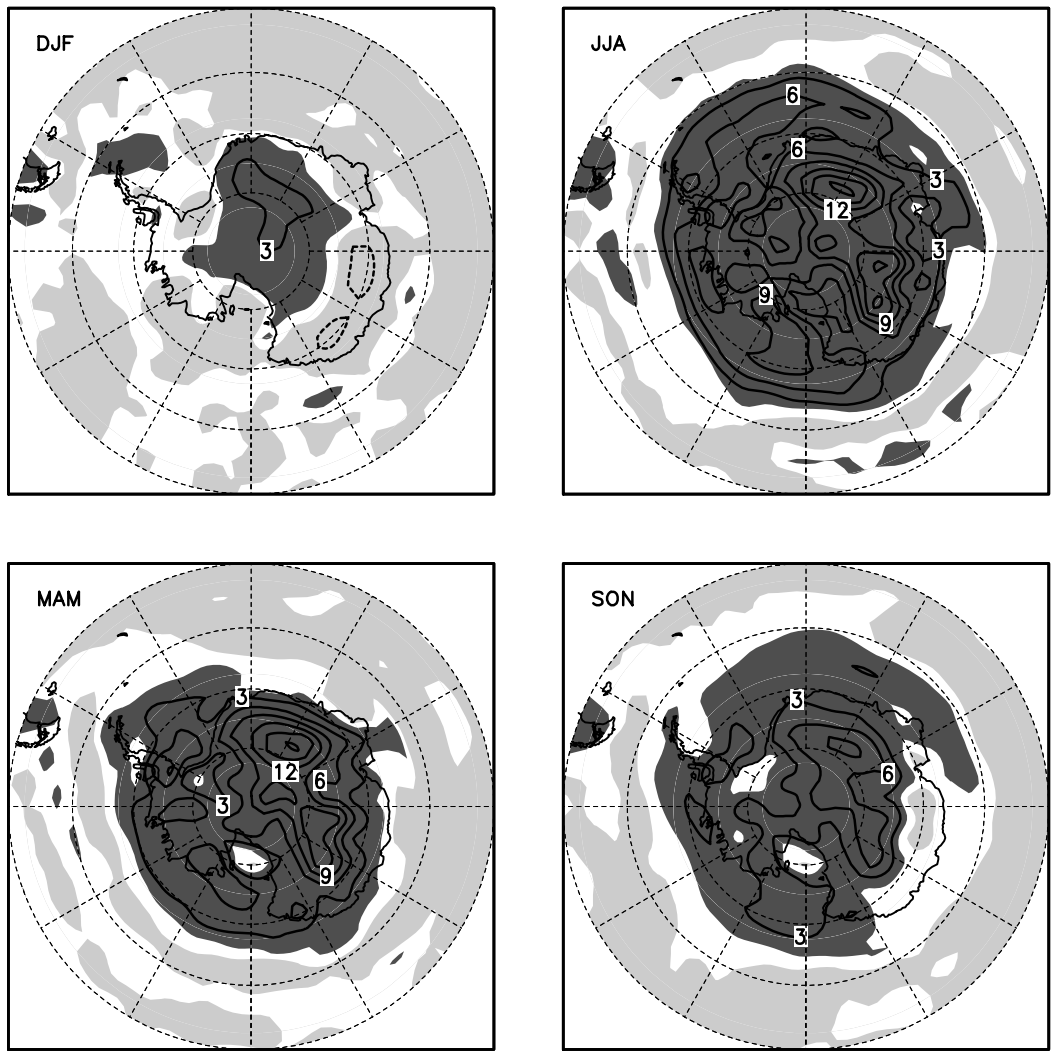

Figura 1a - Diferença sazonal média em ${ }^{\circ} \mathrm{C}$ das climatologias de temperatura em $1000 \mathrm{hPa}$ entre ERA-40 e NNR-1. O intervalo dos contornos é $3^{\circ} \mathrm{C}$. Linhas contínuas são positivas. As áreas com hachuras escuras englobam valores positivos, e as claras, negativos. Dados significativos ao nível de confiança de $95 \%$. A linha de zero foi omitida. 
b) ERA-40 minus NNR-2 T1000
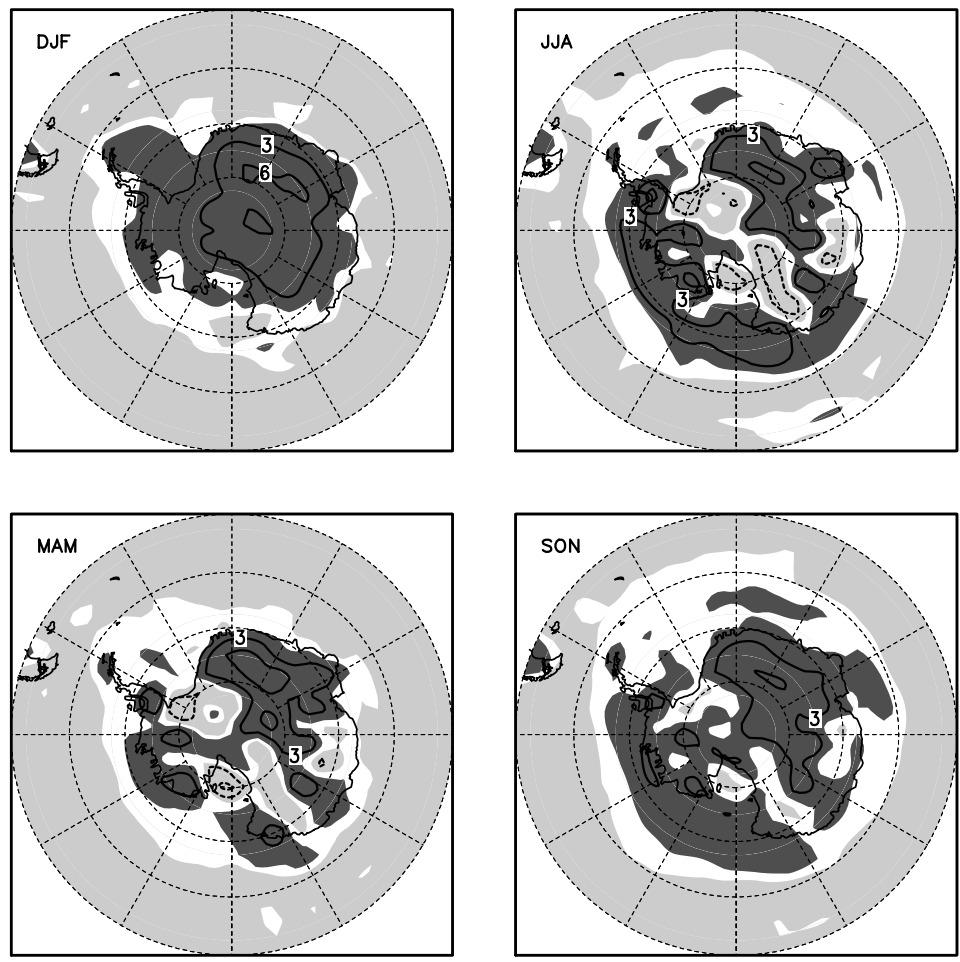

Figura 1b - Como na Fig. 1a, mas para a diferença entre ERA-40 e NNR-2.

c) NNR-2 minus NNR-1 T1000
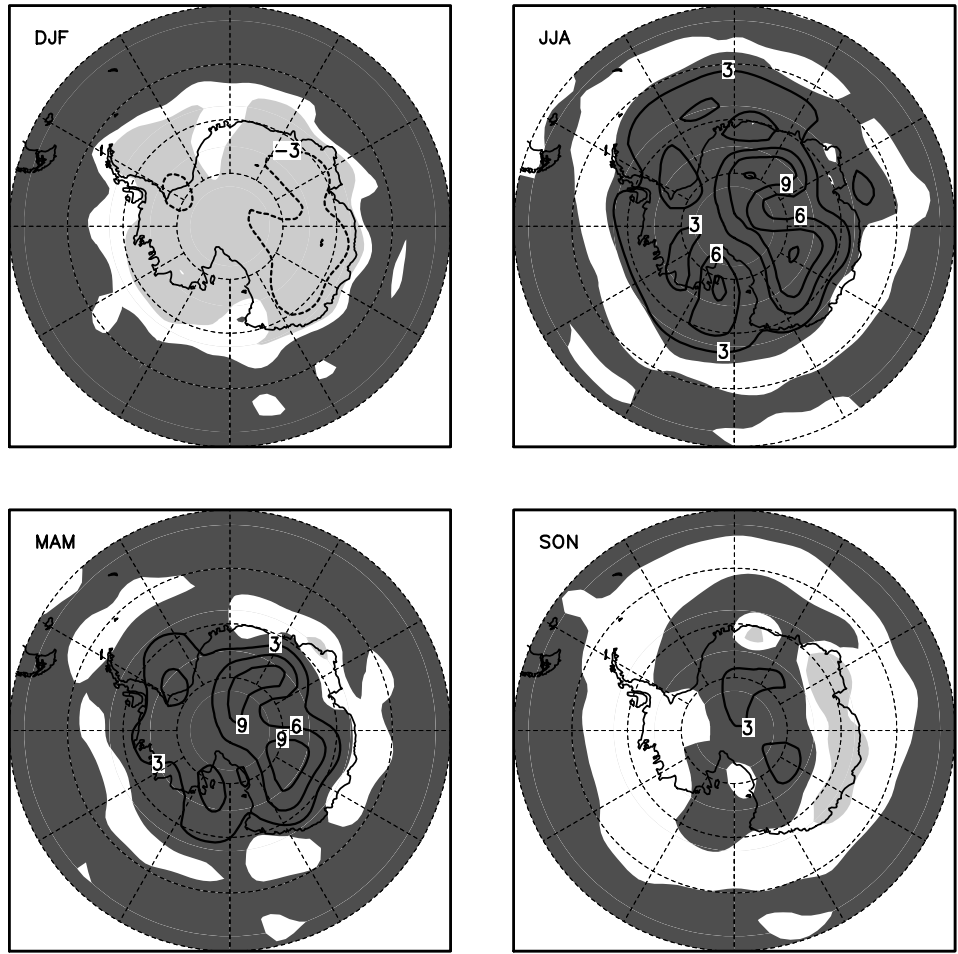

Figura 1c - Como na Fig. 1a, mas para a diferença entre NNR-2 e NNR-1. 
e, como exemplo, incluí-se a temperatura sazonal média do ar para as estações russas de Bellingshausen e Mirny, ambas na linha da costa antártica. Estas estações estão indicadas nos primeiro mapa das Figuras 1a, 1b e 1c. Os cálculos foram feitos a partir das médias mensais obtidas do SCAR-BASREADER (2006) - ver Tabela 1. Para mesmos períodos não devem ser esperadas diferenças significativas entre os valores de temperatura nas estações e os pontos de grade mais próximos destas. Isto porque, os algoritmos de interpolações devem manter os valores da estação em suas localizações geográficas, e a região é essencialmente marítima (ainda que eventualmente congelada), e com pequena variação espacial de temperatura do ar. Em relação às estações, as diferenças foram maiores em Mirny, atingindo $5^{\circ} \mathrm{C}$ no verão, e menores em Bellingshausen, chegando até $1.8^{\circ} \mathrm{C}$ no inverno. As diferenças menores para Bellingshausen na Tabela 1 podem ser explicadas por sua latitude de $\sim 62^{\circ} \mathrm{S}$, a cerca de 4 graus mais ao norte que Mirny, que está em $\sim 66^{\circ} \mathrm{S}$; como vistas nas figuras, as discrepâncias entre as três reanálises se reduzem de sul para norte e tendem a cessar em latitudes próximas de $60^{\circ} \mathrm{S}$.
Entre as razões para as discrepâncias das reanálises, podem-se supor os erros de filtragem de dados em sua ingestão, e o emprego de equações termodinâmicas indevidamente. Para a primeira hipótese, relata-se que se encontrou a PNM mensal com valores em torno de $1600 \mathrm{hPa}$ no conjunto de dados do NNR; estes valores foram corrigidos no NNR após nossa notificação do caso (NCEP, 2006). Quanto às equações, a questão estaria no uso da "atmosfera padrão" na correção da pressão lida nas estações de superfície para outros níveis sobre o continente Antártico. A atmosfera padrão foi revisada em 1976 pela International Civil Aviation Organization (COESA, 1976), ICAO, e é usada globalmente em meteorologia para correção dos dados em altitude para outros níveis. Este padrão é baseado em uma média da atmosfera obtida de rádio-sondages em latitudes médias no hemisfério norte, e representa a atmosfera em um estado permanente ideal, fixo, sob condições moderadas de radiação solar. As estações de superfície no platô antártico apresentam características incomuns, como sua altitude elevada, temperaturas extremamente baixas, pouca umidade, e ausência (ou excesso) de radiação solar, o que faz com que o perfil vertical

a) ERA-40 minus NNR-1 MSLP
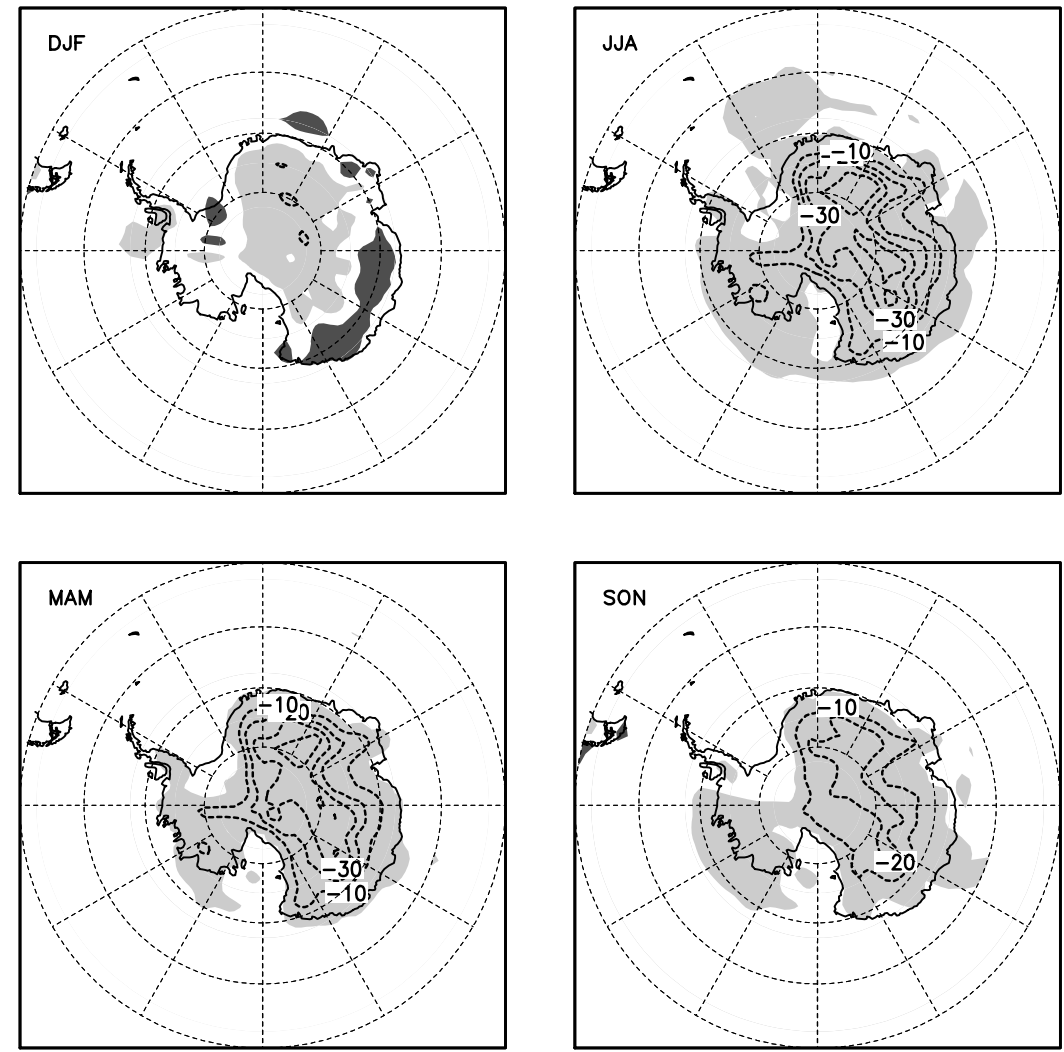

Figura 2a - Diferença sazonal média em hPa das climatologias de PNM entre ERA-40 e NNR-1. O intervalo dos contornos é 10 hPa . As linhas tracejadas indicam valores negativos. As áreas com hachuras escuras englobam valores negativos, e as claras, positivos. Dados significativos ao nível de confiança de $95 \%$. A linha de zero foi omitida. 
b) ERA-40 minus NNR-2 MSLP
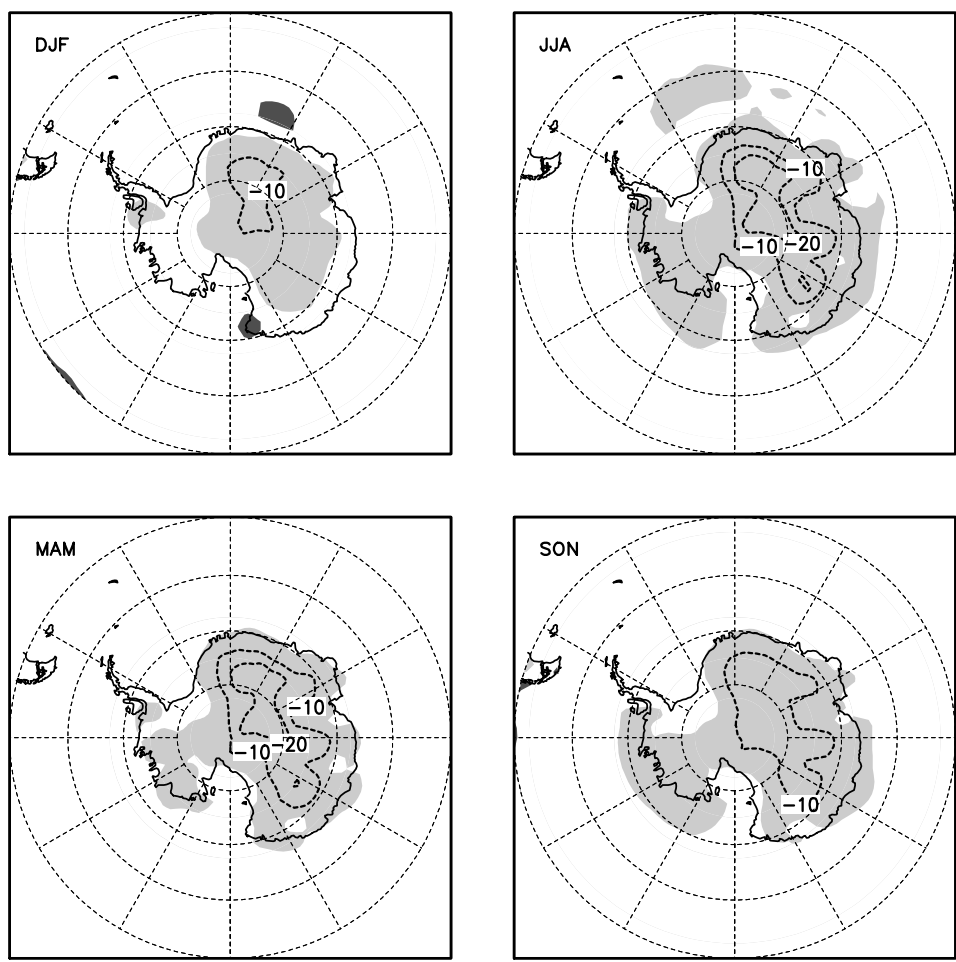

Figura 2b - Como na Fig. 2a, mas para a diferença entre ERA-40 e NNR-2.

c) NNR-2 minus NNR-1 MSLP
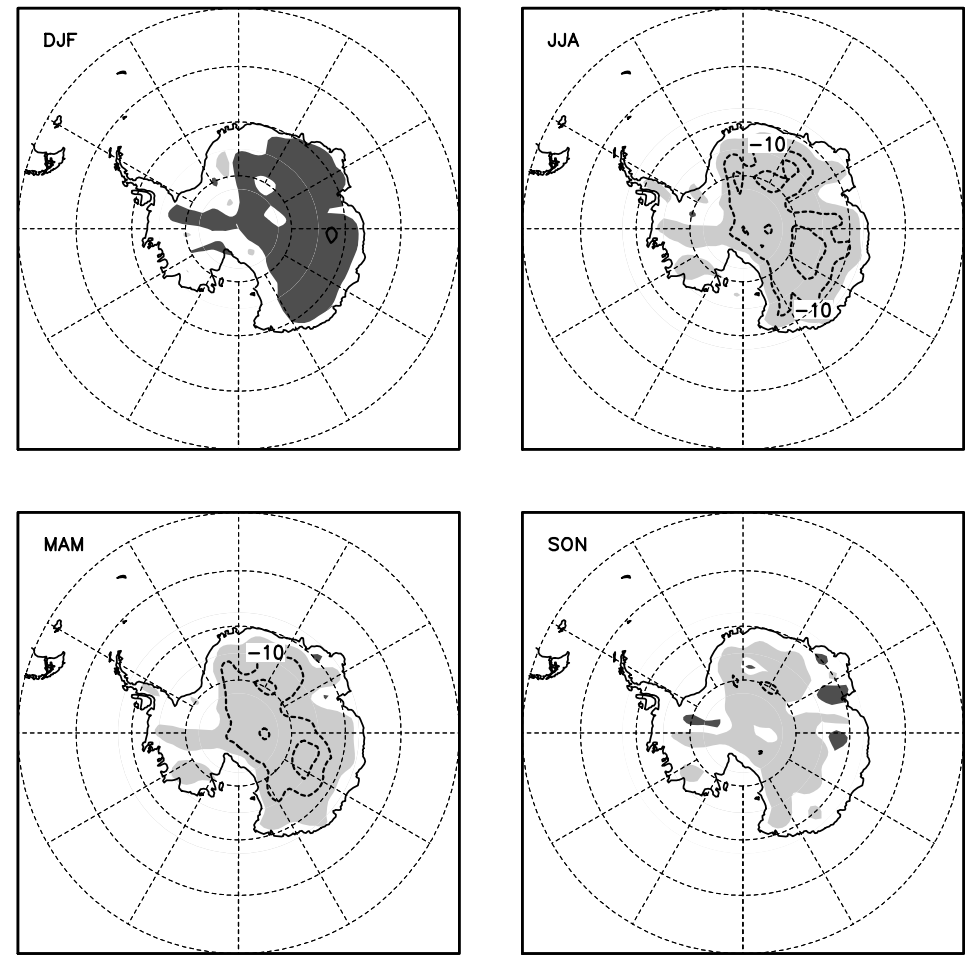

Figura 2c - Como na Fig. 2a, mas para a diferença entre NNR-2 e NNR-1. 
Tabela 1. Comparação sazonal da temperatura do ar à superfície para as estações de Mirny (OMM No. 89592, 66 $33^{\circ}$ 'S \& $093^{\circ} 01^{\prime}$ E, 40 m absl) e Bellingshausen (OMM No. 89050, 62 ${ }^{\circ} 12^{\prime}$ S \& $058^{\circ} 56^{\prime}$ W, 15 m absl) com os dados de reanálises para o período de 1979 a 2000. Os pontos de grade mais pertos para as comparações foram $67^{\circ} 30^{\prime} \mathrm{S}$ e $92^{\circ} 30^{\prime} \mathrm{E}$ a $113 \mathrm{~km}$ e $62^{\circ} 30^{\prime} \mathrm{S}$ e $60^{\circ} \mathrm{W}$ a $64 \mathrm{~km}$, respectivamente. Valores entre parênteses mostram as diferenças em ${ }^{\circ} \mathrm{C}$ em relação aos dados das estações.

\begin{tabular}{l|c|c|c|c|c|c|c|c|}
\cline { 2 - 8 } & Mir. & NNR-1 & NNR-2 & ERA40 & Bell. & NNR-1 & NNR-2 & ERA-40 \\
\cline { 2 - 8 } Summer & $\mathbf{- 3 . 4}$ & 0.9 & -1.3 & -0.8 & $\mathbf{1 . 3}$ & 1.7 & 1.7 & 1.9 \\
(DJF) & & $(4.5)$ & $(2.1)$ & $(2.6)$ & & $(0.4)$ & $(0.4)$ & $(0.6)$ \\
Autumn & $\mathbf{- 1 3 . 4}$ & -13.5 & -12.4 & -12.6 & $\mathbf{- 1 . 6}$ & -1.6 & -1.2 & -0.2 \\
(MAM) & & $(-0.1)$ & $(1.0)$ & $(0.8)$ & & $(0.0)$ & $(0.4)$ & $(1.4)$ \\
Winter & $\mathbf{- 1 6 . 1}$ & -17.7 & -15.8 & -14.5 & $\mathbf{- 5 . 8}$ & -6.4 & -5.6 & -4.0 \\
(JJA) & & $(-1.6)$ & $(0.3)$ & $(1.6)$ & & $(-0.6)$ & $(0.2)$ & $(1.8)$ \\
Spring & $\mathbf{- 1 2 . 4}$ & -10.1 & -11.2 & -10.3 & $\mathbf{- 2 . 8}$ & -2.1 & -2.2 & -1.4 \\
(SON) & & $(2.3)$ & $(1.2)$ & $(2.1)$ & & $(0.7)$ & $(0.6)$ & $(1.4)$ \\
Year & $\mathbf{- 1 1 . 3}$ & -10.1 & -10.2 & -9.6 & $\mathbf{- 2 . 2}$ & -2.1 & -1.8 & -0.9 \\
(J to D) & & $(1.2)$ & $(1.1)$ & $(1.7)$ & & $(0.1)$ & $(0.4)$ & $(1.3)$ \\
\hline
\end{tabular}

na região seja distinto de outras regiões. Esta distinção, com a ausência de equações próprias da região, poderia supostamente causar os erros encontrados nos conjuntos de dados analisados, caso as reanálises utilizem métodos diferentes de correção; infelizmente não foi localizada bibliografia descrevendo este tipo de detalhe nas reanálises. As maiores discrepâncias nas reanálises foram notadas no inverno, quando temperaturas abaixo de $50^{\circ} \mathrm{C}$ negativos no platô são a regra, um fato que reforça nossa suposição. Adicionalmente, considerando a baixa densidade de estações de rádio-sondagem na Antártica, as reanálises deverão gerar campos espaciais distintos se utilizarem métodos de interpolação diferentes.

\section{CONCLUSÕES}

As limitações dos dados de reanálises devem ser consideradas nos estudos climáticos em latitudes antárticas, mesmo após 1979, quando a qualidade da informação é considerada aceitável. Diferenças sazonais na temperatura em $1000 \mathrm{hPa}$, que variam de cerca de $3^{\circ} \mathrm{C}$ a $15^{\circ} \mathrm{C}$, e de até $40 \mathrm{hPa}$ na pressão na superfície, são encontradas entre as reanálises $1 \mathrm{e}$ 2 do NCEP-NCAR, e ECMWF-ERA40. Dados em altitude estão também sujeitos a variações significativas entre os conjuntos de dados. Tais diferenças são uma ordem de grandeza maior que a magnitude do sinal atribuído a tendências climáticas atuais. O problema é conhecido por alguns, e SCAR (2006) listou as diversas sugestões e prioridades para correção dos conjuntos de dados das altas latitudes do hemisfério sul - porém, a conclusão do trabalho é esperada após 2010. A comunidade científica que usa os dados climáticos antárticos e sub-antarticos deve estar a par das limitações dos dados, e de seus efeitos potenciais nas análises de séries temporais e na modelagem. Uma comparação minuciosa das saídas das reanálises para os locais de várias estações de superfície da Antártica poderia fornecer os fatores de correção para as séries climáticas na região.

\section{AGRADECIMENTOS}

Os autores agradecem o apoio financeiro do projeto Proantar número 563880/2005, do CNPq.

\section{REFERÊNCIAS}

BROMWICH, D.H.; FOGT, R.L.; HODGES, K.I.; WALSH, J. A tropospheric assessment of the ERA-40, NCEP, and JRA25 global reanalyses in the polar regions. J. Geophys. Res., v.112, D10111, doi:10.1029/2006JD007859, 2007.

BROMICH, D.H.; FOGT, R.L. Strong trends in the skill of the ERA-40 and NCEP-NCAR Reanalyses in the high and midlatitudes of the southern hemisphere, 1958-2001. J.Climate, v. 17, p.4603-4619, 2004.

COESA, 1976. U.S. Committee on Extension to the Standard Atmosphere. U.S. Standard Atmosphere, 1976. U.S. Government Printing Office, Washington, D.C. 
HINES, K.M.; BROMWICH, D.H.; MARSHALL, G.J. Artificial surface pressure trends in the NCEP-NCAR Reanalysis over the Southern Ocean and Antarctica. J. Climate, v. 13, p.3940-3952, 2000.

KALNAY, E.; KANAMITSU, M.; KISTLER, R.; COLLINS, W.; DEAVEN, D.; GANDIN, L.; IREDELL, M.; SAHA, S.; WHITE, G.; WOOLLEN, J.; ZHU, Y.; CHELLIAH, M.; EBISUZAKI, W.; HIGGINS, W.; JANOWIAK, J.; MO, K.C.; ROPELEWSKI, C.; WANG, J.; LEETMAA, A.; REYNOLDS, R.; JENNE, R.; JOSEPH, D. The NCEP/ NCAR 40-year reanalysis project. Bull. Amer. Meteorol. Soc. , v.77, p. 437-471, 1996.

KANAMITSU, M.; EBISUZAKI, W.; WOOLLEN, J.; YANG, S-K; HNILO, J.J.; FIORINO, M.; POTTER, G. L. NCEPDEO AMIP-II Reanalysis (R-2). Bul. Amer. Meteorol. Soc., v. 83, p. 1631-1643, 2002.

MARSHALL, G.J., Trends in Southern Annular Mode from observations and Reanalyses. J. Climate, v. 16, p. 41344143, 2003.

MARSHALL, G.J.; HARANGOZO, S.A. An appraisal of NCEP-NCAR reanalysis MSLP data variability for climate studies in the South Pacific. Geophys. Res. Lett., v. 27, p. 3057-3060, 2000.

MARSHALL, G.J.; STOTT, P.A.; TURNER, J.; CONNOLLEY, W.M.; KING, J.C.; LACHLAN-COPE, T.A. Causes of exceptional atmospheric circulation changes in the Southern Hemisphere. Geophys. Res. Lett., v.31, p. L14205, doi:10.1029/2004GL019952, 2004.

NOAA-CDC, 2006. Personal communication,

PRESS, W.H.; FLANNERY, B.P.; TEUKOLSKY, S.A.; VETTERLING, W.T. Numerical Recipes: The Art of Scientific Computing. New York: Cambridge University Press, 818 pp. 1986.
SCAR, Report on the SCAR/ClicC/ICPM Workshop on High Latitude Reanalyses, British Antarctic Survey, 10-12/April, 2006, Cambridge, UK. Version of 31/may/2006, 9 pp., at http://www.scar.org/researchgroups/physicalscience/rprt Reanalyses_Wrshp.pdf, 2006.

SCAR-BAS-READER, Scientific Committee on Antarctic Research - Reference Antarctic Data for Environmental Research, British Antarctic Survey, http://www.antarctica. ac.uk/met/READER/, 2006.

SETZER, A.; AQUINO, F.E.; ROMÃO, M.O. Climate tendencies in the South Shetlands: was 1998 a climate divider? Antarctic Peninsula Climatic Variability: Observations, Models, and Plans for IPY Research. National Snow and Ice Data Center at the University of Colorado, p. 28-29, 14-17/maio/2006, 2006.

http://antartica.cptec.inpe.br/ rantar/publicacoes/200605 setzer_aquino_romao_nsidc_apcv_wkshp_ climatetendencies.pdf

UPPALA, S.M.; KÅLLBERG, P.W.; SIMMONS, A.J.; ANDRAE, U.; DA COSTA BECHTOLD, V.; FIORINO, M.; GIBSON, J.K.; HASELER, J.; HERNANDEZ, A.; KELLY, G.A.; LI, X.; ONOGI,K.; SAARINEN, S.; SOKKA, N.; ALLAN, R.P.; ANDERSSON, E.; ARPE, K.; BALMASEDA, M.A.; BELJAARS, A.C.M.; VAN DE BERG, ; L.. BIDLOT, J; BORMANN, N.; CAIRES, S.; CHEVALLIER, F.; DETHOF, A.; DRAGOSAVAC,M.,; FISHER,M. ; FUENTES, M.; HAGEMANN, S.; HÓLM, E.; HOSKINS, B.J.; ISAKSEN,L. ; JANSSEN, P.A.E.M.; JENNE, R.; MCNALLY, A.P.; MAHFOUF, J.-F.; MORCRETTE, J.-J.; RAYNER, N.A.; SAUNDERS, R.W.; SIMON, P.; STERL, A.; . TRENBERTH,K.E; UNTCH, A.; VASILJEVIC, D.; VITERBO, P.; WOOLLEN, J. The ERA-40 re-analysis. Quart. J. R. Meteorol. Soc., v.131, p. 2961-3012, doi:10.1256/qj.04.176. 2005. 